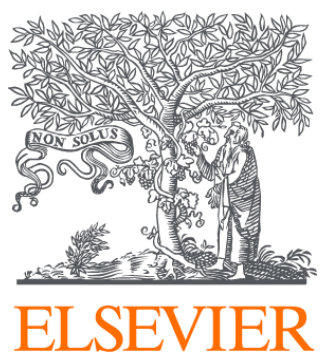

Since January 2020 Elsevier has created a COVID-19 resource centre with free information in English and Mandarin on the novel coronavirus COVID-

19. The COVID-19 resource centre is hosted on Elsevier Connect, the company's public news and information website.

Elsevier hereby grants permission to make all its COVID-19-related research that is available on the COVID-19 resource centre - including this research content - immediately available in PubMed Central and other publicly funded repositories, such as the WHO COVID database with rights for unrestricted research re-use and analyses in any form or by any means with acknowledgement of the original source. These permissions are granted for free by Elsevier for as long as the COVID-19 resource centre remains active. 


\title{
Decreasing mental well-being during the COVID-19 pandemic: A longitudinal study among Danes before and during the pandemic
}

\author{
Lau Caspar Thygesen *, Sanne Pagh Møller, Annette Kjær Ersbøll, Ziggi Ivan Santini, \\ Maj Britt Dahl Nielsen, Morten Klöcker Grønbæk, Ola Ekholm \\ National Institute of Public Health, University of Southern Denmark, Studiestrcede 6, DK-1455, Copenhagen, Denmark
}

\section{A R T I C L E I N F O}

\section{Keywords:}

Mental well-being

Mental health

Panel study

Covid-19

Public health surveillance

Denmark

\begin{abstract}
A B S T R A C T
The COVID-19 pandemic has had a huge impact on people's lives and may influence mental health in the general population. In a unique representative Danish longitudinal study, we examined mental well-being measured just before and during the COVID-19 pandemic. The Danish Health and Wellbeing Survey is the Danish contribution to the European Health Interview Survey. In this study, we included the wave from autumn 2019, which we reinvited in the autumn 2020. The study population consisted of 4,234 persons. The main outcome was mental well-being measured by the Short Warwick-Edinburgh Mental Well-Being Scale (SWEMWBS). Linear and logistic regression models were conducted to evaluate change in SWEMWBS between 2019 and 2020. The SWEMWBS distribution was similar in 2019 and 2020, although the distribution moved to lower scores in 2020 compared to 2019. Mean SWEMWBS decreased significantly from 25.5 in 2019 to 24.6 in 2020 corresponding to a mean change of -1.0 (95\%CI, -1.1 . to -0.8 ). The proportion with low SWEMWBS increased from $16.5 \%$ in 2019 to $20.1 \%$ in 2020 ( $\mathrm{p}<0.001$ ). The mean change was similar for men and women and for different age groups. The most negative development was observed among persons without depression or long-standing illnesses at baseline and among persons with higher educational level. Among persons with depression SWEMWBS increased. As expected, mental well-being significantly decreased in the adult Danish population during the COVID-19 pandemic. However, somewhat more unexpectedly, the decrease was most pronounced among persons without depression or long-standing illnesses and among higher educated groups.
\end{abstract}

\section{Introduction}

The COVID-19 pandemic has a pronounced influence on everyday life and it may have implications for mental health in the general population. The widespread use of quarantine, working and schooling from home, and the consequently social distancing is assumed to be associated with negative psychological effects including post-traumatic stress symptoms, confusion, anger, and infection fears, especially during longer lasting quarantines (Brooks et al., 2020). Furthermore, the crisis will influence living conditions for many persons. The economic consequences of the crisis will be substantial in the short and long run, both on the societal and individual level (Silva et al., 2018).

During 2020, research has increasingly focused on the mental health consequences of COVID-19 (Holmes et al., 2020). Several factors were associated with the decreasing mental health, including the severity of COVID-19 in the individual's home city (Zhang et al., 2020), demographic factors, such as young age (Every-Palmer et al., 2020; Pierce et al., 2020), old age (Ferreira et al., 2021), female sex (Ferreira et al., 2021), and family structure e.g. living with young children (Pierce et al., 2020), socioeconomic factors including job loss (Every-Palmer et al., 2020) and, finally, health factors including poor health status and past diagnosis of mental illness (Every-Palmer et al., 2020).

Previous studies used different measures of life satisfaction, mental health, and symptoms. In a Swiss study, $41.4 \%$ of the participants reported worsened quality of life compared to before the COVID-19 pandemic (Moser et al., 2020) and in a study from New Zealand, 39\% reported low well-being, which was markedly higher than before the pandemic (Every-Palmer et al., 2020). In the United Kingdom, increasing prevalence of mental suffering was observed (Pierce et al., 2020). One study compared well-being during the first months of the pandemic and lockdown in Denmark with a previous representative sample showing that the psychological well-being was negatively

\footnotetext{
* Corresponding author.

E-mail address: lct@sdu.dk (L.C. Thygesen).
} 
affected by the COVID-19 pandemic and more so for females than for males (Sønderskov et al., 2020a). In addition, a multinational study observed a significant decrease in mental well-being using the Short Warwick-Edinburgh Mental Well-Being Scale (SWEMWBS) (Ammar et al., 2020).

The previous studies have shown that mental health decreases during the pandemic based on cross-sectional surveys (Varma et al., 2020; Wang et al., 2020; Zhang et al., 2020), including surveys with questions related to mental health outcomes before the pandemic (Ammar et al., 2020; Ferreira et al., 2021; Moser et al., 2020), and panel studies with repeated measures during the pandemic (Clotworthy et al., 2020; Every-Palmer et al., 2020; Parola et al., 2020; Sønderskov et al., 2020a, b).

In a Danish representative longitudinal study with repeated selfreported data on mental well-being in the general adult population we examined changes in mental well-being in the period from just before the COVID-19 pandemic (autumn 2019) to during the pandemic (autumn 2020). Furthermore, we assessed whether population subgroups experience different changes.

\section{Material and methods}

The Danish Health and Wellbeing Survey is the Danish part of the European Health Interview Survey (EHIS). In this study, we included the wave from autumn 2019, which we re-invited in the autumn of 2020. The design of these surveys is described in detail elsewhere (Rosendahl Jensen et al., 2021).

Briefly, EHIS covers the following topics: Health status (self-rated health, chronic conditions, mental health, accidents, etc.), health determinants (smoking, alcohol consumption, body height and weight, physical activity, dietary habits, etc.), health care utilization (use of different types of health care services), and social and demographic characteristics (marital status, labor market participation, children, household composition, etc.). In the 2019 Danish questionnaire, SWEMWBS was also included (Koushede et al., 2019).

Residents in Denmark have a personal identification number which is used throughout administrative registers and stored in the Civil Registration System (Pedersen, 2011). From the Civil Registration System, 14,000 persons aged 15 years or more were randomly selected and invited to the survey in 2019. Data were collected via a self-administered questionnaire and 6,629 persons (47\%) completed the questionnaire.

Since the survey in late 2019 was carried out just before the COVID19 pandemic reached Europe and Denmark, there was a unique opportunity to examine the longitudinal effects on health and health-related outcomes. We invited all from the 2019 survey, who were still alive and living in Denmark in mid-August 2020, to participate in the followup survey. We excluded persons with specific reasons for non-response in 2019 (e.g. 'hard' refusers, persons with severe cognitive impairment, and persons not understanding Danish). Thus, 13,474 persons aged 16 years or older were invited in 2020. All invited persons were informed of the special nature of the COVID-19 situation, which was reflected in a range of additional questions. The questionnaire focused on the impact of the COVID-19 pandemic on three areas: Physical and mental health, employment and working lives, and health behavior. However, the questionnaire also included questions on areas such as healthcare utilization, social relations, and use of social media. In all, 6,712 persons completed the self-administered questionnaire in 2020, out of which 5,000 had also completed the questionnaire in 2019.

Data in 2019 were collected between 5 September and December 31, 2019. Data in 2020 were collected between 4 September and November 8, 2020. Denmark had a lockdown starting in March 2020 with restricted travel and closing of schools, shops, working places, and cultural institutions with an opening starting in April 2020 in some areas gradually increasing in May and June. In June to August of 2020, only minor restrictions were still in place, but a general request to work from home was never lifted. By August 2020 the number of COVID-19 cases increased and the use of face masks in public indoor areas became mandatory. During September and October 2020, restrictions were reintroduced both regarding travel restrictions and the number of persons that people could engage with.

Regarding economic consequences, the Danish economy decreased in 2020 resulting in increases in unemployment. The Danish GDP decreased by 4 percent in 2020 . The number of unemployed increased during the first half of 2020 but regained the previous level in the autumn 2020.

\subsection{Information included}

\subsubsection{Primary measure}

We measured mental well-being, the outcome of this study, using metric SWEMWBS., where higher scores of SWEMWBS reflect higher well-being (range 7-35). We included both SWEMWBS as a continuous variable and as a binary variable modelling whether SWEMWBS was low representing probable or possible depression or anxiety (scores below 21) (Warwick Medical School, 2021).

\subsubsection{Explanatory variables}

We included several explanatory variables to describe the development in SWEMWBS from 2019 to 2020 . The questions reflected dimensions of demography (sex and age), mental and somatic diseases, and socio-economic factors. Information on all explanatory variables were obtained in 2019:

Demographic factors: Sex and age included in four categories (15-44 years, 45-59, 60-74, and 75+ years).

Current depression was assessed by the eight-item Patient Health Questionnaire depression scale (PHQ-8), which is an established valid diagnostic depression measure also useful in general population surveys (Kroenke et al., 2009). We included PHQ-8 as a binary variable $(\geq 10$ points) representing a clinically relevant depression.

A standard question on longstanding illness or health problem was also included in the questionnaire: 'Do you have any longstanding illness or longstanding health problem (by long-standing we mean illnesses or health problems which have lasted, or are expected to last, for 6 months or more?'). The response categories were 'yes' and 'no'.

Educational level was divided into three categories (elementary school, upper secondary or vocational education, and higher education). Information on educational level was based on data from the Education Register (Jensen and Rasmussen, 2011) where we included highest attained education. Persons with missing information $(n=29,0.7 \%)$ were categorized as elementary school.

Occupational status was included in five categories (employed, unemployed, transfer payment (student compensation or sick leave), pensioner or other). Information was based on the Employment Classification Module (Petersson et al., 2011) on the most important place of employment during 2019.

\subsection{Weighting}

All analyses were weighted to account for non-response in the survey waves. When calculating weights, we estimated the probability of participating and responding to SWEMWBS $(n=4,234)$ among those invited in both waves $(n=13,474)$ by age in five-year categories and sex. The weights were calibrated to sum to the number of observations included $(4,234)$ and had a range of $0.53-3.37$ with an interquartile range of $0.67-1.18$. Younger persons and males had higher weights and middle-aged (55-74 years) had lower weights. All analyses were weighted meaning that the results reflect a population with the same age- and sex-distribution as the invited population. 


\subsection{Statistical analysis}

Descriptive statistics were used to describe those invited and those responding to both SWEMWBS questionnaires in 2019 and 2020. Proportions, means, and medians were calculated. Furthermore, the distribution of each of the seven items of SWEMWBS was reported.

To examine changes in in SWEMWBS from 2019 to 2020, linear and logistic repeated measurements regression models were conducted. In both analyses, the main interest was on whether mean SWEMWBS or odds of low SWEMWBS were different between 2019 and 2020. Furthermore, to evaluate whether the development in SWEMWBS was different between subgroups, the interaction between time and subgroup was evaluated. All analyses were adjusted for sex and age. Age was included as a continuous variable, and as second- and third-degree polynomials. To take account of the correlation due to the repeated measurements on the same person, we estimated generalized estimation equations linear and logistic regression models, respectively, assuming an exchangeable correlation matrix. As sensitivity analyses, the same models were repeated in the non-weighted sample. Furthermore, to evaluate the influence of a ceiling and floor effects of the SWEMWBS scale, we utilized two approaches of the linear regression models. In one, we excluded persons with extreme SWEMWBS scores in 2019 (scores 7 and 35) and, in the other, we used a censored linear regression model (the tobit model).

Analyses were carried out using SAS version 9.4 except tobit analyses performed using $\mathrm{R}$ version 4.0.3 package VGAM.

\section{Ethical aspects}

Participation in the surveys was voluntary which was informed to the invitees. In Denmark, register and questionnaire studies do not require approval by committees on biomedical research ethics according to Danish legislation. The surveys in 2019 and 2020 were approved by SDU Research \& Innovation Organization (RIO). Since 2016, RIO examines and approves all scientific and statistical projects at the University of Southern Denmark according to the Danish Data Protection Regulation.

\section{Results}

Table 1 shows the distribution of the population invited in both 2019 and $2020(n=13,474)$, and the populations with valid SWEMWBS in
2019 ( $\mathrm{n}=6,047 ; 45 \%)$ and $2020(\mathrm{n}=6,712 ; 50 \%)$ and, finally, the analysis population of those with valid SWEMWBS both in 2019 and 2020 ( $n=4,234 ; 31 \%$ ). Compared to the invited population, the populations responding to SWEMWBS in 2019 and 2020 comprise of a higher proportion of females, middle aged, persons with a higher educational level and persons who are occupied and pensioners. The mean and median SWEMWBS are similar in the three groups of respondents.

Mental well-being shows the same distribution both in 2019 and 2020 although the distribution moved to lower well-being in 2020 compared to 2019 (Fig. 1). The figure shows a decrease in the most extreme high category (a score of 35).

The linear regression model shows a significant decrease in mean SWEMWBS from 25.5 in 2019 to 24.6 in 2020 corresponding to a mean change in the regression model of -1.0 (95\%CI, -1.1 to -0.8 ) (Table 2).

When evaluating subgroup specific changes, both sexes and all age groups show similar changes with no significant difference between the subgroups ( $p$-values for interaction of 0.23 and 0.15 ). In the analyses including current depression and long-standing illness, different changes in mean scores in SWEMWBS were observed with strongest decrease in SWEMWBS among persons without a current depression and persons not reporting long-standing illnesses in 2019. For persons with depression in 2019, the mean SWEMWBS increased from 2019 to 2020 (p for interaction below 0.001).

In the analyses of educational level, the decrease in SWEMWBS was stronger for persons with highest educational level compared to lower educational level ( $p$ for interaction of 0.042). In terms of occupational status, the changes in SWEMWBS were strongest for employed and selfemployed persons, but these changes were not significantly different ( $p$ for interaction of 0.15).

The analyses not weighted for non-response are reported in Supplementary Table 1 , which showed similar results. When excluding persons with extreme SWEMWBS scores in 2019 and when performing tobit regression analyses, we found results similar to those reported in Table 2 (not shown).

Supplementary Figure 1 shows the development of each of the seven questions included in SWEMWBS from 2019 to 2020. For all seven questions, a pattern of smaller proportions for the highest positive category combined with higher proportions for the middle categories in 2020 compared to 2019 is seen. Question 6 (I've been feeling close to other people') shows the strongest change with increase in the

Table 1

Baseline descriptives of invited population and populations with valid mental well-being scale (SWEMWBS) in 2019 and/or 2020. Number of observations (percentages) if nothing else noted. Not weighted by non-response weights.

\begin{tabular}{|c|c|c|c|c|}
\hline & Invited in both 2019 and 2020 & Valid SWEMWBS 2019 & Valid SWEMWBS 2020 & Valid SWEMWBS in 2019 and 2020 \\
\hline $\mathbf{N}$ & 13,474 & 6,047 & 6,712 & 4,234 \\
\hline \multicolumn{5}{|l|}{ Sex } \\
\hline Male & $6,524(48)$ & $2,607(43)$ & $2,874(43)$ & $1,765(42)$ \\
\hline \multicolumn{5}{|l|}{ Age } \\
\hline $15-44$ years & $5,943(44)$ & $1,955(32)$ & $2,115(32)$ & $1,136(27)$ \\
\hline 45-59 years & $3,376(25)$ & $1,670(28)$ & $1,929(29)$ & $1,253(30)$ \\
\hline $60-74$ years & $2,809(21)$ & $1,740(29)$ & $1,914(29)$ & $1,416(33)$ \\
\hline Elementary school & $3,685(27)$ & $1,266(21)$ & $1,442(21)$ & $755(18)$ \\
\hline Upper secondary / vocational education & $4,718(35)$ & $2,270(38)$ & 2,608 (39) & $1,668(39)$ \\
\hline Higher education & $4,801(36)$ & $2,448(40)$ & $2,588(39)$ & $1,782(42)$ \\
\hline No information & $270(2)$ & $63(1)$ & $74(1)$ & $29(1)$ \\
\hline \multicolumn{5}{|l|}{ Occupational status } \\
\hline Employed and self-employed & $7,239(54)$ & $3,234(53)$ & $3,656(54)$ & $2,304(54)$ \\
\hline Unemployed & $647(5)$ & $223(4)$ & $244(4)$ & $145(3)$ \\
\hline In 2020 , mean/median & - & - & $24.3 / 24.1$ & $24.6 / 24.1$ \\
\hline
\end{tabular}




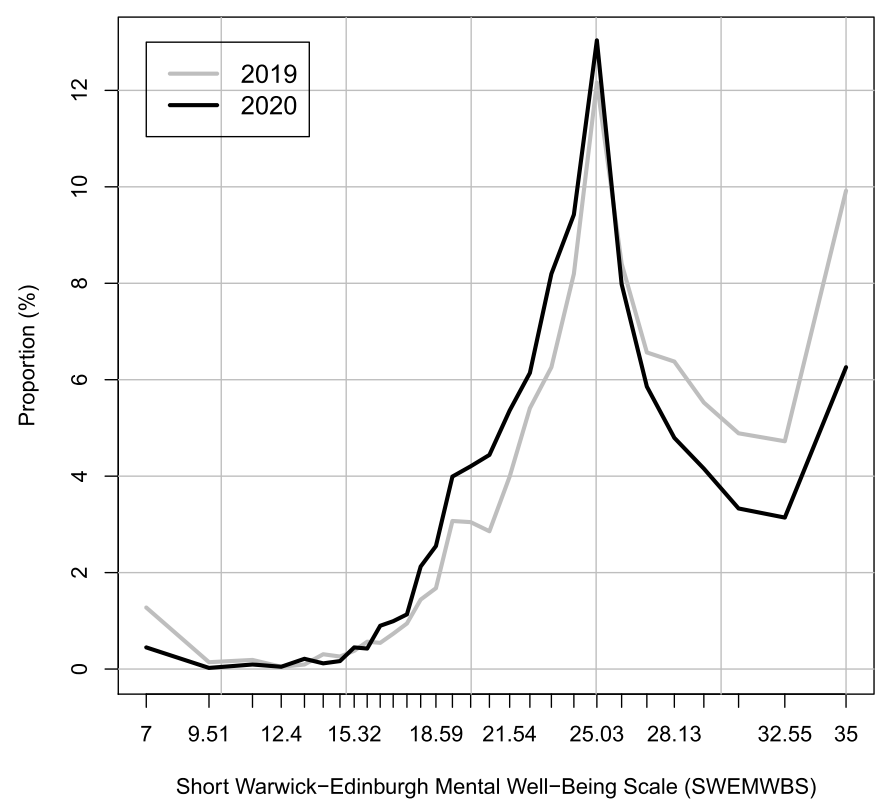

Fig. 1. Distribution of mental well-being (SWEMWBS) in 2019 and 2020.

categories 'rarely' and 'some of the time'.

The logistic regression model evaluating the odds of low mental wellbeing in 2019 and 2020 shows significantly higher odds of low mental well-being in 2020 compared to 2019 ( $\mathrm{p}<0.001$ ). The proportion with low mental well-being is $16.5 \%$ in 2019 and $20.1 \%$ in 2020 (Table 3).

This change is the same among males and females and among the four age groups (p-values for interaction of 0.79 and 0.30 , respectively). The changes are significantly different between persons with current depression and long-standing illness; among persons with current depression the proportion with low mental well-being decreases between 2019 and 2020. Regarding occupational status, there is a significant different development for the five groups (p-value for interaction of 0.031 ) with a decreasing proportion among unemployed persons.

The analyses not weighted for non-response are reported in Supplementary Table 2, which showed similar results.

\section{Discussion}

The aim was to describe the mental well-being in a longitudinal study with self-reported data measured just before and during the COVID-19 pandemic. We observed a significant decrease in mental wellbeing measured by SWEMWBS. This decrease was similar among both sexes and in age groups, but, somewhat surprisingly, was strongest among those without current depression or long-standing illness in 2019. Finally, the decrease was stronger for persons with highest educational level compared to lower educational groups.

The COVID-19 pandemic has pronounced influence on everyday life and may influence mental well-being and living conditions in the general population (Brooks et al., 2020; Silva et al., 2018). We hypothesized that mental well-being decreased during the lockdown rather fast, and that this decrease may be prolonged. The results support this hypothesis as we find a mean decrease of 1.0 in mean SWEMWBS score and an increase in the proportion of people with low mental well-being from $16.5 \%$ to $20.1 \%$ from autumn 2019 to autumn 2020 . The mean decrease corresponds to a minimally important level of change (Warwick Medical School, 2021).

This decrease is in line with other studies reporting decrease in mental health and well-being (Ammar et al., 2020; Every-Palmer et al., 2020; Moser et al., 2020; Pierce et al., 2020). In the only other study using SWEMWBS, a significant decrease from 27.3 to 24.7 (mean change of 2.6) was observed based on questions during the pandemic where the participants were asked to fill out the questionnaire in regards to how they felt 'before' and 'during' home confinement (Ammar et al., 2020).

Table 2

Mental well-being at time 2019 and 2020 and the difference between subgroups among participants with valid SWEMWBS measure in both 2019 and 2020 (n $=4,234$ ). Linear regression model with repeated statement. Weighted by non-response weights.

\begin{tabular}{|c|c|c|c|c|c|}
\hline \multirow[t]{2}{*}{ Variables measured in 2019} & \multirow[b]{2}{*}{$\mathrm{N}$} & \multirow{2}{*}{$\frac{\text { SWEMWBS } 2019}{\text { Mean }(95 \% \mathrm{CI})}$} & \multirow{2}{*}{$\frac{\text { SWEMWBS } 2020}{\text { Mean }(95 \% \mathrm{CI})}$} & \multirow[t]{2}{*}{ Estimate (1) } & \multirow[t]{2}{*}{$\mathrm{p}$-value (2) } \\
\hline & & & & & \\
\hline All & 4,234 & $25.5(25.4-25.7)$ & $24.6(24.4-24.7)$ & $-1.0(-1.1--0.8)$ & $<0.001$ \\
\hline \multicolumn{6}{|l|}{ Sex } \\
\hline Female & $2,183.9$ & $25.1(24.8-25.6)$ & $24.1(23.9-24.3)$ & $-0.2(-0.5-0.1)$ & \\
\hline \multicolumn{6}{|l|}{ Age } \\
\hline $15-44$ years & $1,867.5$ & $24.1(23.9-24.4)$ & $23.4(23.1-23.6)$ & $0.2(-0.2-0.5)$ & 0.15 \\
\hline \multicolumn{6}{|l|}{ Current depression } \\
\hline Yes & 343.3 & $18.7(18.3-19.1)$ & $19.6(19.2-20.1)$ & $2.0(1.4-2.5)$ & $<0.001$ \\
\hline No & 3797.6 & $25.7(25.6-25.9)$ & $24.7(24.6-24.8)$ & 0.0 (ref) & \\
\hline \multicolumn{6}{|l|}{ Long-standing illness } \\
\hline Yes & 1542.8 & $24.2(23.9-24.4)$ & $23.6(23.3-23.8)$ & $0.4(0.0-0.7)$ & 0.024 \\
\hline No & 2666.4 & $25.6(25.4-25.8)$ & $24.6(24.4-24.8)$ & 0.0 (ref) & \\
\hline \multicolumn{6}{|l|}{ Educational level } \\
\hline Unemployed & 161.8 & $20.9(20.2-21.6)$ & $20.7(20.0-21.4)$ & $0.8(0.1-1.5)$ & \\
\hline Transfer payment & 593.5 & $24.0(23.5-24.5)$ & $23.4(22.9-23.9)$ & $0.4(-0.2-0.9)$ & \\
\hline Pensioner & $1,046.4$ & $26.0(25.7-26.4)$ & $25.2(24.9-25.5)$ & $0.2(-0.2-0.6)$ & \\
\hline Other & 69.6 & $25.1(23.7-26.4)$ & $23.9(22.8-25.1)$ & $-0.2(-1.0-0.7)$ & \\
\hline
\end{tabular}

(1) Linear regression adjusted for sex and age. The estimate for all is the difference between 2019 and 2020 . For the stratifying variables, the estimate is the interaction estimate, i.e. the additional effect of the stratifying variable in addition to time and the variable.

(2) The p-value for the analysis of all is the influence of time. For the stratifying variables, the p-value is a test of interaction between time (2019 and 2020 ) and the stratifying variable. 
Table 3

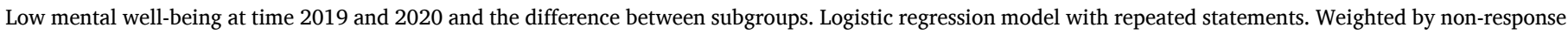
weights.

\begin{tabular}{|c|c|c|c|c|}
\hline \multirow[t]{3}{*}{ Variables } & \multirow[b]{3}{*}{$\mathrm{N}$} & \multicolumn{2}{|c|}{ Low SWEMWBS } & \multirow[b]{3}{*}{ p-value (1) } \\
\hline & & 2019 & 2020 & \\
\hline & & N (\%) & $\mathrm{N}(\%)$ & \\
\hline All & 4,234 & $699.0(16.5)$ & $849.4(20.1)$ & $<0.001$ \\
\hline \multicolumn{5}{|l|}{ Sex } \\
\hline Male & $2,050.1$ & $333.9(16.3)$ & $401.4(19.6)$ & 0.79 \\
\hline Female & $2,183.9$ & $365.2(16.7)$ & $447.9(20.5)$ & \\
\hline \multicolumn{5}{|l|}{ Age } \\
\hline $15-44$ years & $1,867.5$ & $384.2(20.6)$ & $479.4(25.7)$ & 0.30 \\
\hline 45-59 years & $1,060.9$ & $152.1(14.3)$ & $182.7(17.2)$ & \\
\hline $60-74$ years & 882.7 & $82.8(9.4)$ & $107.6(12.2)$ & \\
\hline $75+$ years & 423.0 & 79.9 (18.9) & $79.7(18.8)$ & \\
\hline \multicolumn{5}{|l|}{ Current depression } \\
\hline Yes & 388.5 & $244.9(71.3)$ & $211.2(61.5)$ & $<.0001$ \\
\hline No & $3,770.7$ & $421.2(11.1)$ & $604.5(15.9)$ & \\
\hline \multicolumn{5}{|l|}{ Long-standing illness } \\
\hline Yes & $1,542.8$ & $365.8(23.7)$ & $385.6(25.0)$ & 0.002 \\
\hline No & $2,666.4$ & 329.1 (12.3) & $459.6(17.2)$ & \\
\hline \multicolumn{5}{|l|}{ Educational level } \\
\hline Elementary school & 884.8 & $194.8(22.0)$ & $232.5(26.3)$ & 0.91 \\
\hline Upper secondary/vocational education & $1,481.0$ & $242.9(16.4)$ & $289.8(19.6)$ & \\
\hline Higher education & $1,868.2$ & $261.4(14.0)$ & $327.1(17.5)$ & \\
\hline \multicolumn{5}{|l|}{ Occupational status } \\
\hline Employed and self-employed & $2,362.7$ & $336.6(14.3)$ & $417.1(17.7)$ & 0.031 \\
\hline Unemployed & 161.8 & $85.9(53.1)$ & $78.6(48.6)$ & \\
\hline Transfer payment & 593.5 & $96.5(16.3)$ & $154.0(26.0)$ & \\
\hline Pensioner & $1,046.4$ & $165.3(15.8)$ & $180.8(17.3)$ & \\
\hline Other & 69.6 & $14.7(21.1)$ & $18.8(27.0)$ & \\
\hline
\end{tabular}

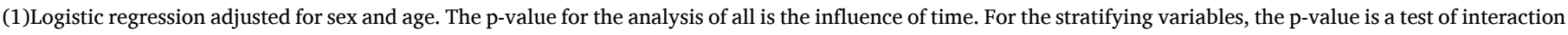
between time (2019 and 2020) and the stratifying variable.

This could result in an overestimation due to recall bias, which is supported by our smaller estimate.

Previous Danish studies also reported negative effects on psychological well-being during the first months of the pandemic (Sønderskov et al., 2020a, b), but afterwards a positive development in the autumn of 2020 (Sønderskov, 2021). In another Danish study, it was reported that worries were stable during the lockdown (Clotworthy et al., 2020). The timing of our survey (4 September to November 8, 2020) seems important in the interpretation of the results since this was a period with re-entry of travel restrictions, limitations on the number of persons people were allowed to engage with, and requirements to use face masks in public indoor areas. Our findings suggest a rather prolonged effect on mental well-being in line with another study with data from autumn 2020 (Sønderskov, 2021). Particularly, our study showed the greatest decrease in mental well-being for the item "I've been feeling close to other people". This would appear to agree with other studies suggesting that loneliness has intensified during the pandemic (Santini and Koyanagi, 2021).

Furthermore, previous studies have reported that some subgroups may be more affected, e.g. younger (Every-Palmer et al., 2020; Pierce et al., 2020) and older (Ferreira et al., 2021), females (Ferreira et al., 2021), persons who experience job loss (Every-Palmer et al., 2020), persons with poor health status and past diagnosis of mental illness (Every-Palmer et al., 2020). Our findings do not support different developments between men and women or different age groups, although younger persons have lower SWEMWBS scores than middle aged persons.

Contrary to other studies, SWEMWBS scores decreased stronger among persons without depression or long-standing illness before COVID-19, while among those with depression, mental well-being increased during the pandemic. In an Australian study, poorer mental health outcomes were reported among those with poor health status or with a previous diagnosis of mental illness (Every-Palmer et al., 2020) and in a study from Bangladesh among persons with asthma, diabetes or cardiovascular diseases, the prevalence of anxiety symptoms and depression symptoms during the COVID-19 pandemic was higher compared to healthy persons (Sayeed et al., 2020). In both studies, no information on pre-COVID-19 mental health or symptoms was reported. A similar approach to ours was conducted in a study from the Netherlands of adults with bipolar disorder, where information on psychiatric symptoms and loneliness from 2017-18 were compared to information during the COVID-19 pandemic (Orhan et al., 2021). The study reported that participants experienced less psychiatric symptoms during COVID-19 compared to before the pandemic, which seems to be consistent with our results.

Although the COVID-19 pandemic is generally reported to be associated with decreases in mental health, it should not be assumed that decreases are inevitable. In the current study, we observed a general decrease in mental well-being among the general adult population, but an increase among those with depression. Similarly, we observed a general decrease in mental well-being overall, except for the lowest SWEMWBS scores, where there appeared to be an improvement (Fig. 1). Although this finding may seem counter-intuitive, there are reasons why people with low mental well-being or depression may fare better during the pandemic. First, it is possible that the pandemic conditions (social distancing measures and the risk of infection) have alleviated some social norms and reduced expectations to socially interact. Contrary to the general response to increased isolation, it may be that people with depression are better equipped to cope with isolation, since they are familiar with social withdrawal. Furthermore, closer contact and more time spent with immediate family members could have benefitted those suffering from depression (Bruining et al., 2020). Other research has documented that other crises have been associated with improved social functioning (Mancini, 2020), which may be explained by acute stress in a crisis stimulating affiliate, cooperative and trusting behavior, which could potentially benefit those with depression (Mancini, 2019).

Our findings indicate a rather prolonged negative effect on mental well-being in the general population and especially loneliness has intensified during the pandemic. Further, some subgroups that might usually be considered vulnerable (i.e individuals with depression at 
baseline) did not experience deteriorating mental health, but rather the non-clinical population saw a decline. Overall, these findings support general population-based interventions to reduce the negative mental health impacts of COVID-19. This may include providing reliable COVID-19 information to alleviate anxiety and fear and interventions to increase physical activity and coping skills (Safieh et al., 2021). Recent meta-analytic research provides guidance into the most effective interventions to promote mental well-being and to prevent low mental well-being through various universal or targeted approaches including intersectoral collaborations (Santini et al., 2020; van Agteren et al., 2021). The results of our study show that it might be beneficial to specifically focus on interventions that limit loneliness and promotes alternative forms of social interaction. In times of physical distancing, quarantine, and restrictions on social contacts, digital interventions may play an important role in improving public mental health. Digital mental health interventions can be used to deliver mental health treatment and preventive services for people with mental health problems or to deliver health promotion and prevention efforts targeting high-risk individuals, subpopulations, and entire populations (Rauschenberg et al., 2021). Whereas most digital interventions focus on prevention and treatment, there is some evidence supporting mental health promotion, e.g. to increase physical activity and coping skills and reduce alcohol consumption, stress, and loneliness (Ebert et al., 2019; Rauschenberg et al., 2021).

Our study had several strengths, including a rather large representative sample with repeated measures of mental well-being. The prospective collection of data is a main strength of our data, which makes it possible to compare data collected during the COVID-19 pandemic with data collected just before the outbreak among the same persons. This comparison provides unique information, which is only available in few other studies (Kwong et al., 2020; Orhan et al., 2021; Pierce et al., 2020), while most other studies have used one or more measurements during the COVID-19 pandemic (Ammar et al., 2020; Clotworthy et al., 2020; Every-Palmer et al., 2020; Ferreira et al., 2021; Moser et al., 2020; Parola et al., 2020; Sønderskov et al., 2020a, b; Varma et al., 2020; Wang et al., 2020; Zhang et al., 2020). The main advantage is that we can take account of any pre-COVID-19 suboptimal mental well-being, when evaluating the effect of the COVID-19 pandemic. This means that we can separate the effects of each predictor, e.g. how depression is associated with mental well-being, from the effect of the COVID-19 pandemic, i.e. comparison of mental well-being before and during the pandemic, and, finally, evaluate the COVID-19 effect for each predictor level. Furthermore, the data collection was conducted during autumn both years limiting the influence of seasonal variations in mental health.

Our study may be influenced by selection bias since the response proportion was rather low (2019: 47\%; 2020: 50\%), and because we only included persons who completed the questionnaire in both 2019 and 2020. To limit the influence of selection bias, we weighted the analyses by response weights based on age- and sex-distribution of the invited sample.

The instrument used to measure mental well-being (SWEMWBS) has shown satisfactory validity in a Danish setting and is recommended to be used in epidemiological studies (Koushede et al., 2019). Demographic and socioeconomic variables were obtained from nationwide registers with satisfactory validity (Jensen and Rasmussen, 2011; Petersson et al., 2011). Information on depression was based on PHQ-8, which is a valid measure of current depression in population-based studies (Kroenke et al., 2009). The question on long-standing illness is a standard item used in numerous surveys and is part of the Minimum European Health Module. For all variables, we only included information obtained before the COVID-19 pandemic ensuring a clear temporal ordering.

Another element, when including repeated measures, is the influence of regression towards the mean and ceiling and floor effects. The regression model used in this paper (repeated measures model focusing on the interaction between subgroup and time) assumes that the subgroups we study, e.g. persons with depression or without depression, do reflect distinct populations before the pandemic (Van Breukelen, 2006), i.e. that the mean SWEMWBS is different in 2019. This assumption seems reasonable, and we do not think our results are strongly influenced by regression towards the mean. We evaluated the influence of ceiling and floor effects by performing tobit regression and excluded persons with extreme SWEMWBS scores in 2019. Both analyses support the main results.

\section{Conclusion}

In conclusion, our study reports a significant decrease in mental wellbeing during the COVID-19 pandemic in a general population-based study based on prospectively collected data. This decrease was surprisingly most pronounced among persons without depression or longstanding illnesses and among higher educated groups. Contrary to expectations, people with depression appeared to fare better during the pandemic as compared to prior to the outbreak.

\section{Declaration of competing interest}

All authors have no conflicts of interest to declare that are relevant to the content of this article.

\section{Acknowledgement}

We thank Katrine Strandberg-Larsen for valuable discussions of the analytical model and interpretation of results.

\section{Appendix A. Supplementary data}

Supplementary data to this article can be found online at https://doi. org/10.1016/j.jpsychires.2021.09.035.

\section{Data sharing}

Data are linked to administrative registers and can only be accessed through affiliation with University of Southern Denmark. The authors welcome any contacts regarding collaboration. The questionnaires (in Danish) are accessible at https://www.sdu.dk/da/sif/forskning/p rojekter/betydningen_af_covid_19_krisen.

\section{Authors' contributions}

LCT, SPM, AKE, MBDN, MKG and OE designed the study. LCT analyzed the data and drafted the first version of the manuscript. All authors interpreted the data and contributed to the development of the manuscript including critical revision and drafting for important intellectual content. All authors agree to be accountable for all aspects of the work in ensuring that questions related to the accuracy or integrity of any part of the work are appropriately investigated and resolved.

\section{Role of funding source}

The project was financed by a grant by Velliv Foreningen (grant number 20-0438). The funding source did not have any role in the analyses and interpretation of the results of this article.

\section{References \\ Ammar, A., Mueller, P., Trabelsi, K., Chtourou, H., Boukhris, O., Masmoudi, L., Bouaziz, B., Brach, M., Schmicker, M., Bentlage, E., How, D., Ahmed, M., Aloui, A., Hammouda, O., Paineiras-Domingos, L.L., Braakman-Jansen, A., Wrede, C., Bastoni, S., Pernambuco, C.S., Mataruna-Dos-Santos, L.J., Taheri, M., Irandoust, K., Khacharem, A., Bragazzi, N.L., Adrian Washif, J., Glenn, J.M., Bott, N.T., Gargouri, F., Chaari, L., Batatia, H., Khoshnami, S.C., Samara, E., Zisi, V., Sankar, P., Ahmed, W.N., Ali, G.M., Abdelkarim, O., Jarraya, M., El Abed, K., Romdhani, M., Souissi, N., Van Gemert-Pijnen, L., Bailey, S.J., Moalla, W., Gomez-Raja, J., Epstein, M., Sanderman, R., Schulz, S., Jerg, A., Al-Horani, R., Mansi, T., Jmail, M.,}


Barbosa, F., Ferreira-Santos, F., Simunic, B., Pisot, R., Gaggioli, A., Zmijewski, P., Steinacker, J.M., Strahler, J., Riemann, L., Riemann, B.L., Mueller, N., Chamari, K, Driss, T., Hoekelmann, A., Consortium, E.-C., 2020. Psychological consequences of COVID-19 home confinement: the ECLB-COVID19 multicenter study. PloS One 15 (11), e0240204.

Brooks, S.K., Webster, R.K., Smith, L.E., Woodland, L., Wessely, S., Greenberg, N., Rubin, G.J., 2020. The psychological impact of quarantine and how to reduce it: rapid review of the evidence. Lancet 395 (10227), 912-920.

Bruining, H., Bartels, M., Polderman, T.J.C., Popma, A., 2020. COVID-19 and child and adolescent psychiatry: an unexpected blessing for part of our population? Eur. Child Adolesc. Psychiatr. 30 (7), 1139-1140.

Clotworthy, A., Dissing, A.S., Nguyen, T.L., Jensen, A.K., Andersen, T.O., Bilsteen, J.F., Elsenburg, L.K., Keller, A., Kusumastuti, S., Mathisen, J., Mehta, A., Pinot de Moira, A., Rod, M.H., Skovdal, M., Strandberg-Larsen, K., Tapager, I.W., Varga, T.V., Vinther, J.L., Xu, T., Hoeyer, K., Hulvej Rod, N., 2020. 'Standing together - at a distance': documenting changes in mental-health indicators in Denmark during the COVID-19 pandemic. Scand. J. Publ. Health 49 (1), 79-87. https://doi.org/10.1177/ 1403494820956445.

Ebert, D.D., Harrer, M., Apolinario-Hagen, J., Baumeister, H., 2019. Digital interventions for mental disorders: key features, efficacy, and potential for artificial intelligence applications. Adv. Exp. Med. Biol. 1192, 583-627.

Every-Palmer, S., Jenkins, M., Gendall, P., Hoek, J., Beaglehole, B., Bell, C., Williman, J., Rapsey, C., Stanley, J., 2020. Psychological distress, anxiety, family violence, suicidality, and wellbeing in New Zealand during the COVID-19 lockdown: a crosssectional study. PloS One 15 (11), e0241658.

Ferreira, L.N., Pereira, L.N., da Fe Bras, M., Ilchuk, K., 2021. Quality of life under the COVID-19 quarantine. Qual. Life Res. 30 (5), 1389-1405.

Holmes, E.A., O'Connor, R.C., Perry, V.H., Tracey, I., Wessely, S., Arseneault, L., Ballard, C., Christensen, H., Cohen Silver, R., Everall, I., Ford, T., John, A., Kabir, T., King, K., Madan, I., Michie, S., Przybylski, A.K., Shafran, R., Sweeney, A., Worthman, C.M., Yardley, L., Cowan, K., Cope, C., Hotopf, M., Bullmore, E., 2020. Multidisciplinary research priorities for the COVID-19 pandemic: a call for action for mental health science. Lancet Psychiatry 7 (6), 547-560.

Jensen, V.M., Rasmussen, A.W., 2011. Danish education registers. Scand. J. Publ. Health 39 (7 Suppl), 91-94.

Koushede, V., Lasgaard, M., Hinrichsen, C., Meilstrup, C., Nielsen, L., Rayce, S.B., TorresSahli, M., Gudmundsdottir, D.G., Stewart-Brown, S., Santini, Z.I., 2019. Measuring mental well-being in Denmark: validation of the original and short version of the Warwick-Edinburgh mental well-being scale (WEMWBS and SWEMWBS) and crosscultural comparison across four European settings. Psychiatry Res. 271, 502-509.

Kroenke, K., Strine, T.W., Spitzer, R.L., Williams, J.B., Berry, J.T., Mokdad, A.H., 2009 The PHQ-8 as a measure of current depression in the general population. J. Affect. Disord. 114, 163-173, 1-3.

Kwong, A.S.F., Pearson, R.M., Adams, M.J., Northstone, K., Tilling, K., Smith, D., FawnsRitchie, C., Bould, H., Warne, N., Zammit, S., Gunnell, D.J., Moran, P.A., Micali, N., Reichenberg, A., Hickman, M., Rai, D., Haworth, S., Campbell, A., Altschul, D., Flaig, R., McIntosh, A.M., Lawlor, D.A., Porteous, D., Timpson, N.J., 2020. Mental health before and during the COVID-19 pandemic in two longitudinal UK population cohorts. Br. J. Psychiatry 1-10. https://doi.org/10.1192/bjp.2020.242.

Mancini, A.D., 2019. When acute adversity improves psychological health: a socialcontextual framework. Psychol. Rev. 126 (4), 486-505.

Mancini, A.D., 2020. Heterogeneous mental health consequences of COVID-19: costs and benefits. Psychol Trauma 12, S15-S16.

Moser, A., Carlander, M., Wieser, S., Hammig, O., Puhan, M.A., Hoglinger, M., 2020. The COVID-19 Social Monitor longitudinal online panel: real-time monitoring of social and public health consequences of the COVID-19 emergency in Switzerland. PloS One 15 (11), e0242129.

Orhan, M., Korten, N., Paans, N., de Walle, B., Kupka, R., van Oppen, P., Kok, A., Sonnenberg, C., Schouws, S., Dols, A., 2021. Psychiatric symptoms during the COVID-19 outbreak in older adults with bipolar disorder. Int. J. Geriatr. Psychiatry 36 (6), 892-900.

Parola, A., Rossi, A., Tessitore, F., Troisi, G., Mannarini, S., 2020. Mental health through the COVID-19 quarantine: a growth curve analysis on Italian young adults. Front. Psychol. 11, 567484.
Pedersen, C.B., 2011. The Danish Civil registration System. Scand. J. Publ. Health 39 (7 Suppl), 22-25.

Petersson, F., Baadsgaard, M., Thygesen, L.C., 2011. Danish registers on personal labour market affiliation. Scand. J. Publ. Health 39 (7 Suppl), 95-98.

Pierce, M., Hope, H., Ford, T., Hatch, S., Hotopf, M., John, A., Kontopantelis, E., Webb, R., Wessely, S., McManus, S., Abel, K.M., 2020. Mental health before and during the COVID-19 pandemic: a longitudinal probability sample survey of the UK population. Lancet Psychiatry 7 (10), 883-892.

Rauschenberg, C., Schick, A., Hirjak, D., Seidler, A., Paetzold, I., Apfelbacher, C., RiedelHeller, S.G., Reininghaus, U., 2021. Evidence synthesis of digital interventions to mitigate the negative impact of the COVID-19 pandemic on public mental health: rapid meta-review. J. Med. Internet Res. 23 (3), e23365.

Rosendahl Jensen, H.A., Thygesen, L.C., Moller, S.P., Dahl Nielsen, M.B., Ersboll, A.K., Ekholm, O., 2021. The Danish Health and Wellbeing Survey: study design, response proportion and respondent characteristics. Scand. J. Publ. Health. https://doi.org/ $10.1177 / 14034948211022429$ online ahead of print.

Safieh, J., Broughan, J., McCombe, G., McCarthy, N., Frawley, T., Guerandel, A., Lambert, J.S., Cullen, W., 2021. Interventions to optimise mental health outcomes during the COVID-19 pandemic: a scoping review. Int J Ment Health Addict 1-22. https://doi.org/10.1007/s11469-021-00558-3.

Santini, Z.I., Koyanagi, A., 2021. Loneliness and its association with depressed mood, anxiety symptoms, and sleep problems in Europe during the COVID-19 pandemic. Acta Neuropsychiatr. 33 (3), 160-163.

Santini, Z.I., Stougaard, S., Koyanagi, A., Ersboll, A.K., Nielsen, L., Hinrichsen, C., Madsen, K.R., Meilstrup, C., Stewart-Brown, S., Koushede, V., 2020. Predictors of high and low mental well-being and common mental disorders: findings from a Danish population-based study. Eur J Public Health 30 (3), 532-538.

Sayeed, A., Kundu, S., Al Banna, M.H., Christopher, E., Hasan, M.T., Begum, M.R., Chowdhury, S., Khan, M.S.I., 2020. Mental health outcomes of adults with comorbidity and chronic diseases during the COVID-19 pandemic: a matched casecontrol study. Psychiatr. Danub. 32, 491-498, 3-4.

Silva, M., Resurreccion, D.M., Antunes, A., Frasquilho, D., Cardoso, G., 2018. Impact of economic crises on mental health care: a systematic review. Epidemiol. Psychiatr. Sci. 29, e7.

Sønderskov, 2021. Variation in psychological well-being and symptoms of anxiety and depression during the COVID-19 pandemic: Results from a 3-wave panel survey. Acta Neuropsychiatr. 33 (3), 156-159.

Sønderskov, K.M., Dinesen, P.T., Santini, Z.I., Ostergaard, S.D., 2020a. The depressive state of Denmark during the COVID-19 pandemic. Acta Neuropsychiatr. 32 (4), $226-228$.

Sønderskov, K.M., Dinesen, P.T., Santini, Z.I., Ostergaard, S.D., 2020b. Increased psychological well-being after the apex of the COVID-19 pandemic. Acta Neuropsychiatr. 32 (5), 277-279.

van Agteren, J., Iasiello, M., Lo, L., Bartholomaeus, J., Kopsaftis, Z., Carey, M., Kyrios, M., 2021. A systematic review and meta-analysis of psychological interventions to improve mental wellbeing. Nat. Hum. Behav. 5 (5), 631-652.

Van Breukelen, G.J., 2006. ANCOVA versus change from baseline: more power in randomized studies, more bias in nonrandomized studies [corrected]. J. Clin. Epidemiol. 59 (9), 920-925.

Varma, P., Junge, M., Meaklim, H., Jackson, M.L., 2020. Younger people are more vulnerable to stress, anxiety and depression during COVID-19 pandemic: a global cross-sectional survey. Prog. Neuro-Psychopharmacol. Biol. Psychiatry 110236. https://doi.org/10.1016/j.pnpbp.2020.110236.

Wang, C., Pan, R., Wan, X., Tan, Y., Xu, L., Ho, C.S., Ho, R.C., 2020. Immediate psychological responses and associated factors during the initial stage of the 2019 coronavirus disease (COVID-19) epidemic among the general population in China. Int. J. Environ. Res. Public Health 17 (5), 1729.

Warwick Medical School, 2021. Collect, Score, Analyse and Interpret WEMWBS. https ://warwick.ac.uk/fac/sci/med/research/platform/wemwbs/using/howto. (Accessed 22 April 2021). Accessed.

Zhang, S.X., Wang, Y., Rauch, A., Wei, F., 2020. Unprecedented disruption of lives and work: health, distress and life satisfaction of working adults in China one month into the COVID-19 outbreak. Psychiatry Res 288, 112958. 